Auch eine Fallzahlsteigerung im laufenden Quartal führt nur über diesen Weg zu einem - sicherlich nur mäßigen finanziell verbesserten Ergebnis, jedoch mit der Perspektive einer Budgetanhebung im Folgejahr. Dies ist nach dem neuen Beschluss des Bewertungsausschusses zum 1.7.2010 aber nicht mehr sicher, denn neben der schon im aktuellen Quartal praktizierten Fallzahlbegrenzung kann auch im Folgejahr zusätzlich eine Begrenzung des Fallzahlzuwachses vorgenommen werden.

\section{MMW Kommentar}

Auch wenn Vertragsärztin und Vertragsarzt nach dem 1.7.2010 schon vier Wochen vor Quartalsbeginn ihr Quartalsbudget nun sogar noch genauer kennen, ist die Perspektive, bei einem höheren Arbeitsaufwand kein höheres Honorar mehr erzielen zu können, nicht gerade motivierend. Umso mehr sollte die Praxis künftig einen Tätigkeitsschwerpunkt dort legen, wo Mehrarbeit noch vergütet wird. Das sind zunächst die verbliebenen extrabudgetären Leistungen. Hier gibt es insbesondere bei der Prävention noch erhebliche Umsatzreserven.

Immer wertvoller werden auch die unterschiedlichen Formen der Berufsausübungsgemeinschaften (BAG) wie Gemeinschaftspraxis oder MVZ. Besonders die Möglichkeit der Gründung einer ortsübergreifenden BAG kann selbst im laufenden Quartal durch die dort vorgesehenen Zuschläge zu einer Umsatzanhebung führen. Bei so einem Zusammenschluss ist kein größerer organisatorischer Aufwand notwendig, bei fachgleicher Konstellation aber ein unmittelbarer Umsatzanstieg um 10\% die Folge. Zuletzt gilt es auch noch, den gesetzlichen Auftrag zu beachten. Leistungen in der GKV sind exakt definiert. Der Patient kann nur diese Leistungen erhalten. Will er mehr, handelt es sich um individuelle Gesundheitsleistungen (IGeL), die privat zu liquidieren sind.

\title{
Vorsicht bei BtM-Rezepten in Gemeinschaftspraxen!
}

- In der Betäubungsmittel-Verschreibungsverordnung (BtMVV) ist festgelegt, wie Ärzte Betäubungsmittel (BtM) verordnen und was sie dabei beachten müssen. In $\S 8$ der BtMVV ist geregelt, dass Betäubungsmittel-Rezepte auf Anforderung von der Bundesopiumstelle des Bundesinstituts für Arzneimittel und Medizinprodukte an den einzelnen Arzt ausgegeben werden. Diese Rezepte enthalten die individuelle siebenstellige BtMNummer des berechtigten Arztes, das Ausgabedatum und die laufende Rezeptnummer.

Die BtM-Nummer wird personenbezogen und somit arztbezogen erteilt. In Gemeinschaftspraxen oder auch im MVZ bedeutet dies, dass jeder Arzt seine eigenen personenbezogenen BtM-Rezepte verwendet und eigene Nachweise zum Verbleib der BtM-Rezepte führen muss.
Sofern der verschreibende Arzt bei solchen Verordnungen den Kassenstempel der Gemeinschaftspraxis verwendet, muss er auf der Verordnung (BtM-Rezept) zusätzlich seinen Namen vermerken.

\section{MMW Kommentar}

Lediglich im Vertretungsfall kann der Arzt ausnahmsweise sein BtM-Rezept auf einen anderen Arzt übertragen. Auf dem BtM-Rezept ist die Vertretung entsprechend kenntlich zu machen oder zu vermerken, und zwar mit der Angabe ,in Vertretung“ bzw. „i.V.“. Zum Praxisstempel des Arztes, der vertreten wird, muss in diesen Fällen der Name des vertretenden Arztes hinzugefügt werden. Ein lückenloser Nachweis über den Verbleib der BtMRezepte muss aber auch in solchen Vertretungsfällen gesichert sein.

\section{Praxis-Pkw ohne Fahrtenbuch}

\begin{abstract}
— Für die private Nutzung eines betrieblichen Kraftfahrzeugs werden vom Finanzamt für jeden Kalendermonat $1 \%$ des inländischen Listenpreises zum Zeitpunkt der Erstzulassung zuzüglich der Kosten für Sonderausstattungen einschließlich der Umsatzsteuer geltend gemacht. Für eine auch private Nutzung des Dienstwagens spricht nach der Rechtsprechung aufgrund der allgemeinen Lebenserfahrung der Beweis des ersten Anscheins.
\end{abstract}

\section{MMW Kommentar}

Dieser Anscheinsbeweis kann jedoch durch einen Gegenbeweis entkräftet werden. Hierzu bedarf es allerdings nicht des Beweises des Gegenteils. Es genügt viel- mehr, dass ein Sachverhalt dargelegt wird, der die Möglichkeit eines anderen als des der allgemeinen Erfahrung entsprechenden Geschehensablaufs ergibt. Die 1\%-Regel kommt nämlich dann nicht zur Anwendung, wenn der Steuerpflichtige den ihm überlassenen Firmenwagen ausschließlich für berufliche Fahrten nutzt. Stehen einem Steuerpflichtigen und seiner Familie ausreichend private Fahrzeuge zur Verfügung, kann nach der Auffassung des Finanzgerichts Niedersachsen in seinem Urteil vom 29.8.2009 von einer - zumindest nahezu - ausschließlich beruflichen Nutzung ausgegangen werden. Dies reicht nach der Auffassung des Gerichts aus, dem Steuerpflichtigen keinen Nutzungsvorteil zuzurechnen. 